\title{
AAAI-08 and IAAI-08 Conferences Provide Focal Point for AI
}

\author{
Sara Reese Hedberg
}

- This summer's AAAI Conference on Artificial Intelligence (AAAI-08) and its sister Conference on Innovative Applications of AI (IAAI-08) continued their long tradition of being a focal point of AI. This year's conferences were held in Chicago at the Hyatt Regency McCormick Place, July 13-17, 2008. The multidimensional conference offerings included nine invited talks, 251 technical papers, 22 innovative applications of AI papers, three competitions (poker, AI video, and general game playing), three special tracks (AI and the web, integrated intelligence, and physically grounded AI), 15 tutorials, 15 workshops, and 11 intelligent system demonstrations, as well as a number of awards, a doctoral consortium, student poster session and programs, and a vendor exhibit. This translated into a plethora of choices for the 921 conference attendees. An additional 175 people exclusively attended the tutorials, workshops, or exhibit.
$\Omega_{\text {hi }}$ sister Conference on Innovative Applications of AI (IAAI08) continued their long tradition of being a focal point of AI. "The annual AAAI conference is a nexus for exchanging the latest ideas in the field," according to Eric Horvitz, president of AAAI and a research manager at Microsoft Research. This year's conferences were held in Chicago at the Hyatt Regency McCormick Place, July 13-17, 2008.

The multidimensional conference offerings included nine invited talks, 251 technical papers, 22 innovative applications of AI papers, three competitions (poker, AI video, and general game playing), three special tracks (AI and the web, integrated intelligence, and physically grounded AI), 15 tutorials, 15 workshops, and 11 intelligent system demonstrations, as well as a number of awards, a doctoral consortium, student poster session and programs, and a vendor exhibit. This translated into a plethora of choices for the 921 conference attendees. An additional 175 people exclusively attended the tutorials, workshops, or exhibit.

\section{Highly Distinguished Invited Speakers}

Perhaps one of the true litmus tests of any conference is the caliber of the invited speakers. The AAAI-08 and IAAI-08 conferences brought 


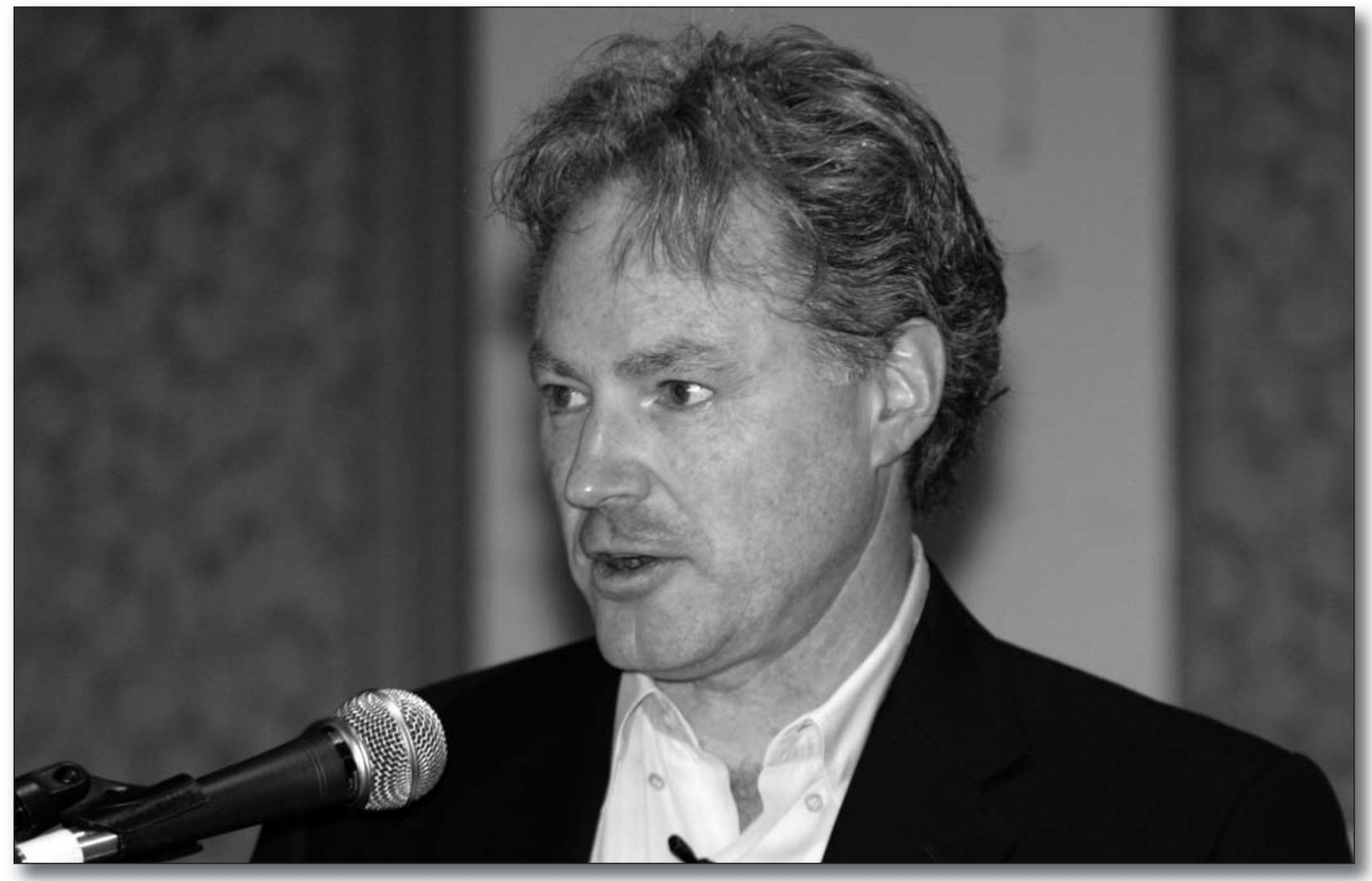

Eric Horvitz Delivers His AAAI Presidential Address at AAAI-08.

together nine distinguished speakers including Eric Horvitz (AAAI president and research manager at Microsoft Research) who gave his AAAI presidential address, "Artificial Intelligence in the Open World." 1

Chris Urmson (Carnegie Mellon University), a leading member of the DARPA Urban Grand Challenge winning team, described the race and winning vehicle in his talk, "Boss, the Urban Challenge, and the Promise of Autonomous Driving," while Mark Newman (University of Michigan) described some of the study of networks across disciplines such as mathematics, sociology, computer science, physics, biology, and so on and presented promising new research in machine learning for network analysis in his talk, "Making Sense of Complex Networks." David Haussler (University of California, Santa Cruz) traced the growing understanding of the human genome in his talk, "100 Million Years of Evolutionary History of the Human Genome."

The distinguished Robert S. Englemore Memorial Award Lecture was delivered by Kenneth Ford (Florida Institute for Human and Machine Cognition). In his lecture, "Toward Cognitive Prostheses," Ford discussed human-centered computing to amplify human cognition and perception.

In other invited talks, Stuart Russell (University of California, Berkeley) examined what has been achieved in AI and what remains to be done to reach the goals imagined by the early AI pioneers ("What Is to Be Done?"), Alexei A. Efros (Carnegie Mellon University) discussed how to enable computers to reason about a scene from a photograph ("From Images to Scenes: Using Lots of Data to Infer Geometric, Photometric, and Semantic Scene Properties from a Single Image"), and Lillian Lee (Cornell University) covered work in analyzing opinions expressed in so- cial computing networks ("Sense and Sensibility: Sentiment Analysis, Opinion Mining, and the Computational Treatment of Subjective Language"), while Seth C. Goldstein (Carnegie Mellon University) discussed revolutionary work in self-reconfiguring programmable matter composed of ensembles of submillimeter robots in his talk, "Realizing Claytronics: A Challenge for AI."

\section{Big Changes Come to Mobile Robot Events}

The 2008 mobile robot events saw a significant departure from the past 16 years of the Mobile Robot Competition and Exhibition. Instead of the popular competition, which has pushed the envelope of mobile robotics since its inception, this year was host to a Robot Workshop and Exhibition.

The workshop afforded an opportu- 
nity to reflect on what has been achieved in mobile robotics and to discuss possible future directions to move the science forward. Distinguished researchers from 15 leading U.S. universities (including Stanford, the Massachusetts Institute of Technology, University of Illinois at Chicago, and Carnegie Mellon), three companies (Microsoft Research, Boeing Phantom Works, and Hanson Robotics), as well as program directors from the Defense Advanced Research Projects Agency (DARPA) and the National Science Foundation (NSF) discussed current research as well as their visions for the future of robots. The focus was on the emerging realm of robots and creativity, and mobility and manipulation.

The robot exhibition was a fascinating amalgamation of cutting-edge experimental robots such as a humanoid robot that extemporaneously danced to music, the first improvisational percussionist robot, a cuddly stuffed-animallike robot initially being used to study early childhood social development, an assistive robot that opens doors and elevators it has never seen before, and a snakelike articulated robot that climbed up willing attendees' legs (protective jeans required.)

\section{Competitions Fuel Innovations}

"AAAI has continued to promote challenging competitions that energize researchers and fuel innovations," Eric Horvitz says of this year's competitions, "and these matches come to a head at the conference." This year's matches included competitions in computer poker, general game playing, and AI videos. "Such challenges can help to elucidate core principles of intelligence," he concluded.

The AAAI computer poker competition, now in its third year, showcased state-of-the-art intelligent programs for playing poker. Recently, poker has become an important challenge problem for AI because, unlike chess, players do not know what cards the other players have, hence the information is "imperfect." Thus traditional AI gameplaying techniques do not apply, and novel approaches are needed. AAAI's competition continues to be the pre-

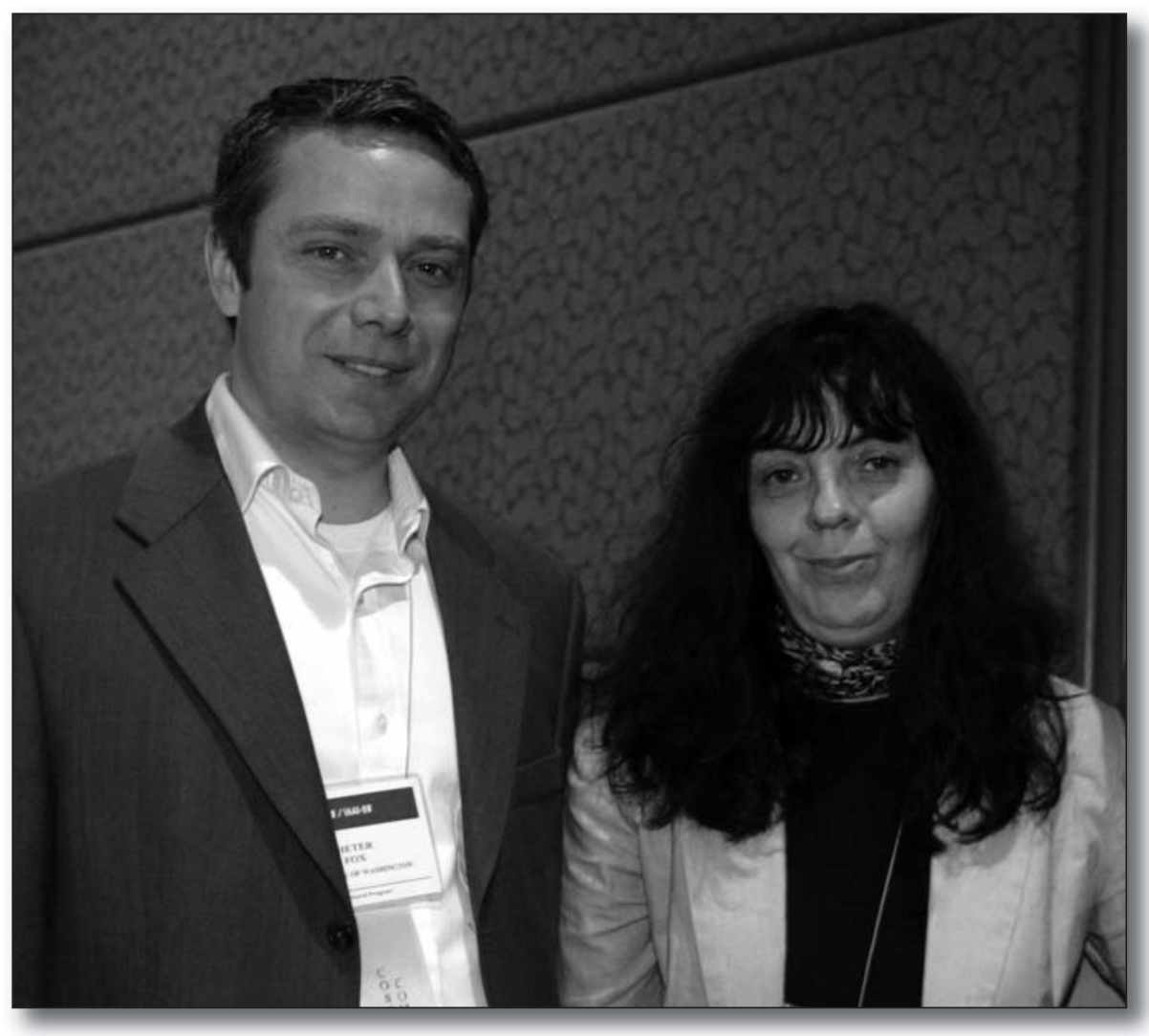

AAAI-08 Technical Program Cochairs Dieter Fox and Carla Gomes.

miere venue for demonstrating current programs.

The bar was raised significantly this year for two-player matches, along with the addition of a multiplayer competition. "This year there was a great turnout and a high level of competition," said Andrew Gilpin, the competition chair. "The 2008 competition had 19 entries from 12 research groups hailing from six countries. Almost all of the entries played at a high level and included all of the top performing programs from previous years (although they have each been improved significantly). The entries this year improved significantly over the previous years' entries. Interestingly, the winning program of the six-player competition was based on research performed several years ago, so it is clear there is still a huge amount of research ground to be covered in multiplayer poker." 2 The next competition is planned for AAAI-09 in Atlanta, Georgia.

Now in its fourth year, the General
Game Playing Competition continues to push the edges of this vein of research. Unlike specialized game players such as the much-touted Deep Blue, the IBM program that beat world chess champion Garry Kasparov in 1997, a general game player accepts game descriptions at run time and can play the game without human intervention. While this line of research is inherently interesting, it is already showing practical applicability in areas such as business process management, e-commerce, and military operations.

In 2008, the competition challenged the general game players by comparing their performance in a variety of previously unseen games. Competition began in May 2008 with the championship round held in Chicago during the conference with eight programs competing representing six countries. "There was stronger play than in previous years," notes Eric Schkufza, the competition's cochair. "There were fun new games for the fi- 

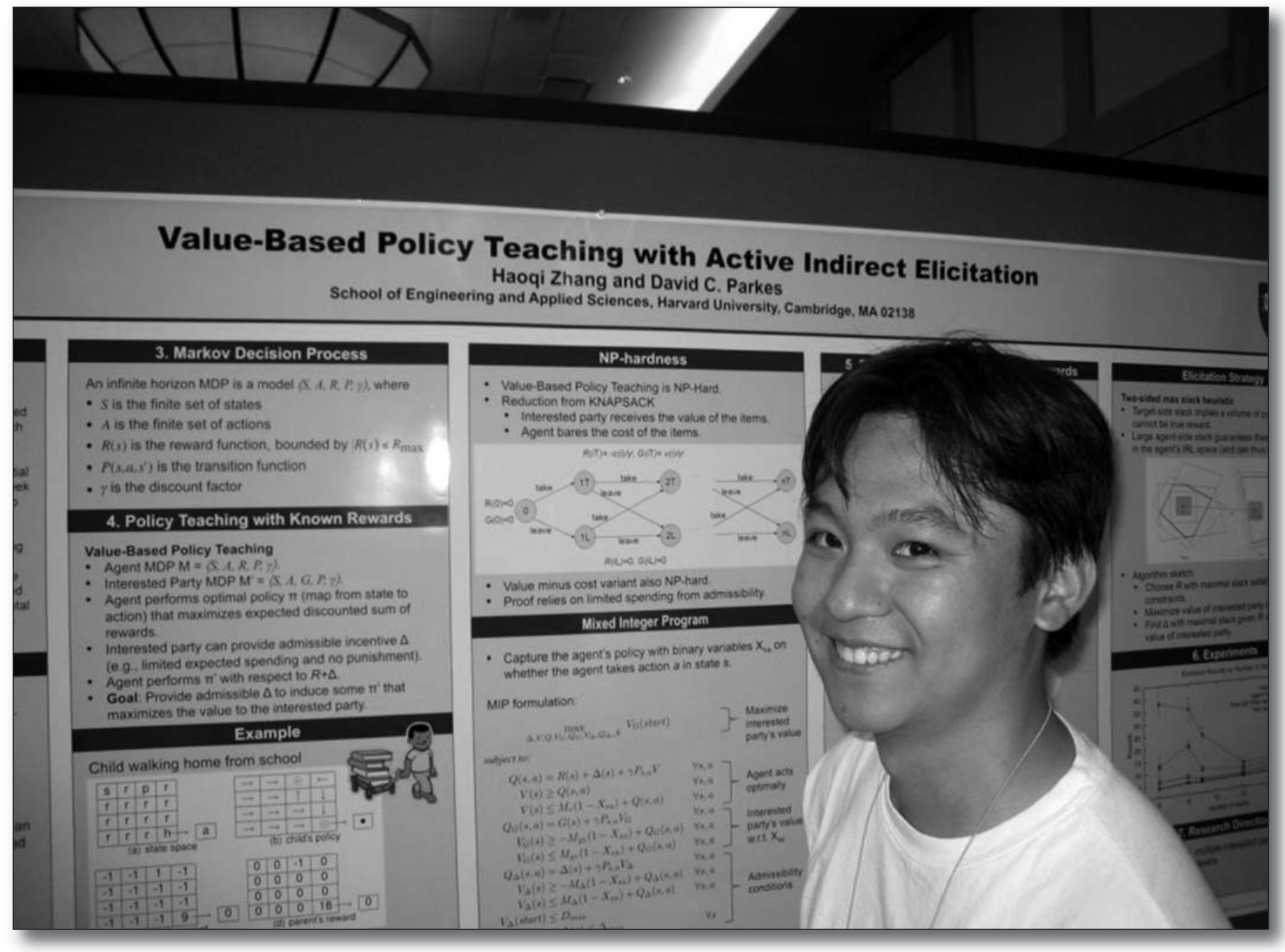

The Poster Session was Well Attended.

nal rounds of competition designed to highlight how general the players are; for instance, tic-tac-chess-checkersfour." 3 The fifth General Game Playing Competition is planned for the 2009 IJCAI conference (cosponsored by AAAI) in Pasadena this coming summer.

Back for its second year, the lively AI video competition entertained and educated while documenting 36 exciting AI projects. This competition, the brainchild of cochairs David Aha and Sebastian Thrun, is exploring new ways to document progress in the field. "The motivation for this competition," explains Aha, "is to encourage the creation and dissemination of videos related to AI research and practice so as to interest prospective stu- dents to consider entering the field of AI."

While going about the serious business of the competition, this group had some good old fashioned fun. "Several of the videos had something fun in them," says Aha. "Take, for example, the best narrative video by David Wilson (University of North Carolina). His video, CBR Noir, is a 1940s-styled whodunit flick centered on a stereotypical private eye character's attempt to solve a murder case. He narrated it in character, focused it on introducing and exemplifying the case-based reasoning problem-solving methodology, and mentioned no less than 27 names of famous case-based reasoning systems, all in horrible puns, throughout the video. It's a mas- terpiece. (We'd love to encourage the creation of similar ones for all areas of AI.)"

This year's entries ranged from a video of bioinspired swarming flying robots to another documenting a system that helps art experts authenticate alleged Van Gogh paintings. ${ }^{4}$ Plans are underway for next summer's competition. Lights, action, camera anyone?

\section{Innovative Applications of AI}

For 20 years, the Innovative Applications of Artificial Intelligence (IAAI) Conference has recognized high-impact computer applications deployed in the real world that utilize AI in nov- 


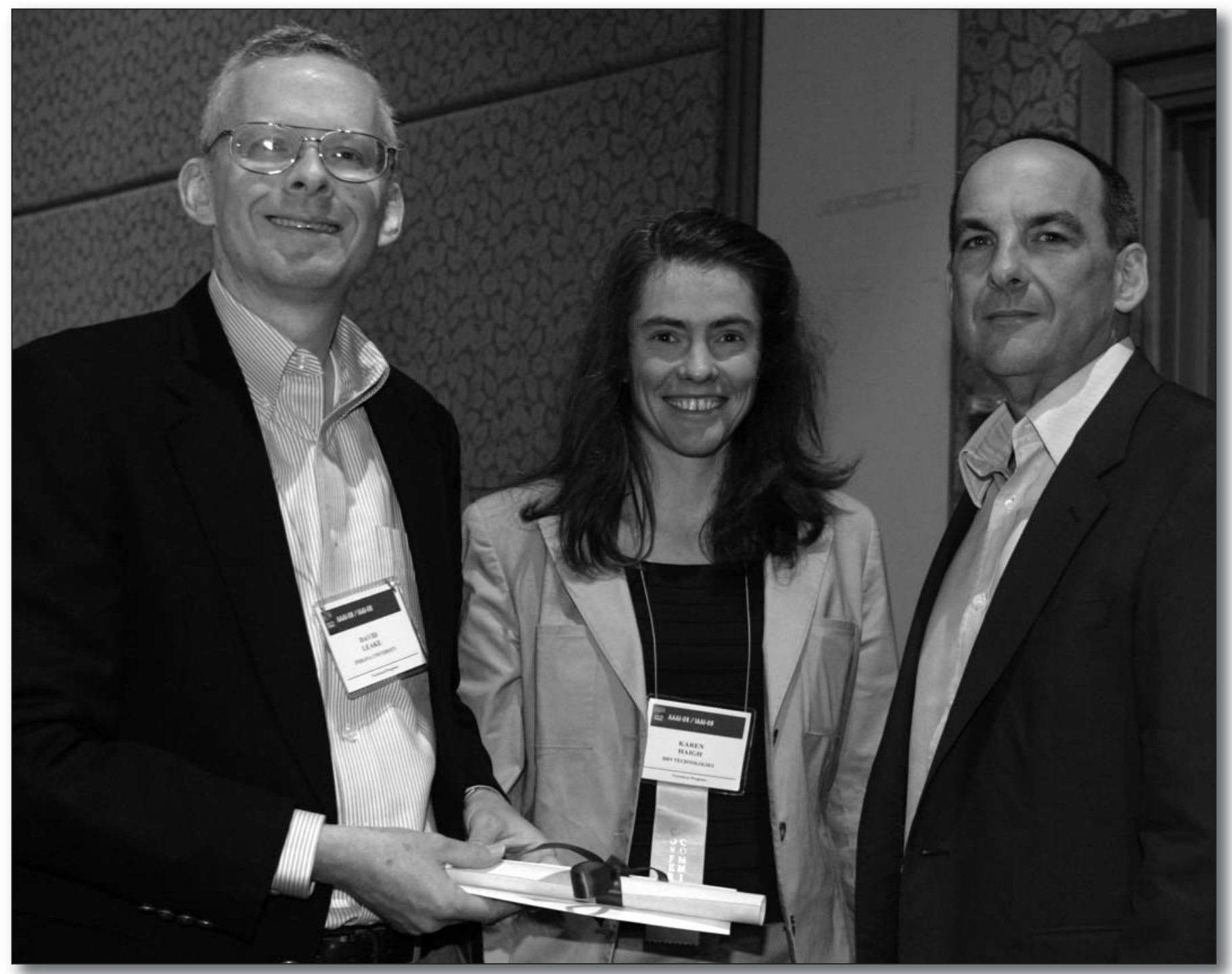

Ken Ford (right) Receives the Robert Engelmore Award from AI Magazine Editor David Leake (left), and IAAI Cochair Karen Haigh.

el ways and have a significant economic return on the investment costs required to develop and deliver the system. To study this legacy of IAAI award-winning applications is to see the evolution of trailblazing AI systems from rare stand-alone applications running on LISP machines to highly ubiquitous applications threaded through the fabric of modern-day life.

Several years ago, the IAAI organizers expanded the scope of the conference to include not only fully deployed applications, but also emerging innovative applications under development that show promising early results. IAAI-08's 22 papers included 4 award-winning deployed applications and 18 emerging applications. Together, they constitute a current sampling of the myriad ways that AI techniques are being harnessed to solve a wide range of problems and issues around the globe. (See the sidebar: "TwentyTwo Far-Reaching IAAI Applications Putting AI to Work around the Globe")

\section{Far-Reaching IAAI Applications Putting AI to Work around the Globe}

The Innovative Applications of Artificial Intelligence Conference honors high-impact real-world applications that utilize AI in novel ways and have a significant economic return on the investment costs required to develop and deliver the system or that are under development and show significant promise. In 2008, the number of selected papers from outside the United States grew to reflect the increasing globalization of AI and our AAAI organization. Topics convered in 2008 included aerospace, computing and networks, education, the environment and earth sciences, medical and health care, and resource scheduling and allocation. 


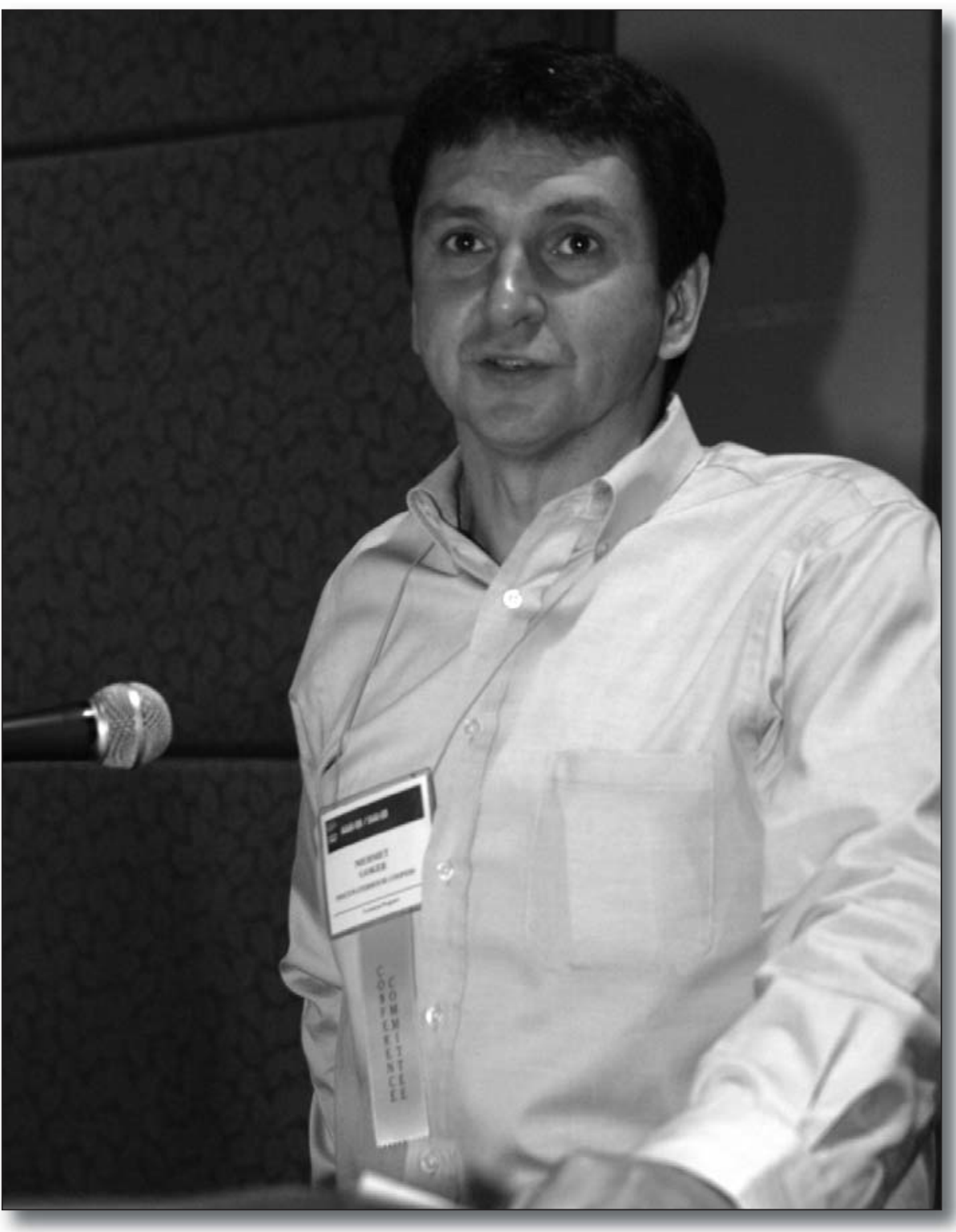

IAAI Chair Mehmet Goker.

\section{Aerospace}

Electrical power systems in aerospace vehicles support critical subsystems such as avionics and life support. NASA Ames Research Center and the University of California Los Angeles presented "Diagnosing Faults in Electrical Power Systems for Spacecraft and Aircraft," a system that provides a diagnostic capability including a highlevel specification language that supports autogeneration of Bayesian networks.

\section{Computing and Networks}

Six IAAI presentations were concerned with computing and networks.
Wake Forest University presented "In-the-Dark Network Traffic Classification Using Support Vector Machine," a system that improves network management by classifying network packet traffic.

The University of Maryland presented "Finding Ontological Correspondences for a Domain-Independent Natural Language Dialog Agent," a system that offers an intelligent speech-based dialog interface for various devices (such as PDA, GPS, and hospital service robots).

BT (UK), 4C, and UCC (Ireland) presented "Personalization of Telecommunications Services as Combinatorial Optimisation," a system that allows users to select and compose telecommunications network features while automatically resolving conflicts.

Knexus Research Corp., CDM Technologies, and the Naval Research Laboratory presented "Enabling the Interoperability of Large-Scale Legacy Systems," a system that addresses the problem of achieving interoperability of disparate large-scale legacy relational databases across client military organizations. It employs an intelligent mapping toolkit using a distributed "federation" of mapping agents, casebased similarity assessment, and learning to semiautomatically acquire domain specific lexicons and thesauri.

\section{Education}

In the area of education, the IAAI conference had three presentations. Peking University (China) presented "A Case Study of AI Application on Language Instruction: CSIEC." Computer Simulation in Educational Communication (CSIEC) is an intelligent web-based human-computer system for English instruction for students in China using natural language processing. Currently there are more than 30,000 registered users.

Alelo, Inc., presented "Tactical Language and Culture Training Systems: Using Artificial Intelligence to Teach Foreign Languages and Cultures," a system that helps users quickly acquire communication skills in foreign languages and cultures through an interactive game format. Between 20,000 and 50,000 learners worldwide, largely U.S. soldiers, have used the 
courses. A pilot Chinese course for college and high school Chinese programs is under development, as well as a pilot Cherokee game intended to help preserve Native American language and culture.

The University of North Carolina presented "The Law of Choice and the Decision Not to Decide," a system that helps parents choose a public school based on the parents' preferences and the students' needs, interest, abilities, and talents.

\section{Environment and Earth Sciences}

There were four applications in the environment and earth sciences area. Air turbulence prediction is critical to airplane safety. The current automated turbulence forecasting system, funded by the Federal Aviation Administration and used by the National Oceanic and Atmospheric Administration's Aviation Weather Center, is based largely on subjective observations reported by pilots coupled with largescale quantitative atmospheric data. The National Center for Atmospheric Research and the University of Colorado at Boulder presented "Application of Artificial Intelligence to Operational Real-Time Clear-Air Turbulence Prediction," a system that applies AI techniques to take advantage of the better and more plentiful source of turbulence data that has become recently available from aircraft sensors with promising results.

NASA Ames Research Center (Moffett Field, CA) presented "Learning to Improve Earth Observation Flight Planning," a system that integrates machine learning with planning and data visualization for the management of mobile sensors for Earth science investigations related to understanding changes to the Earth's ecosystem (such as climate change or pollution). The system supports missions that combine observations from heterogeneous ground, airborne, and spaceborne sensors that must be managed in concert.

NASA Ames Research Center, Goddard Space Flight Center, and the Aerospace Corporation jointly presented "Local Search for Optimal Global Map Generation Using Mid-

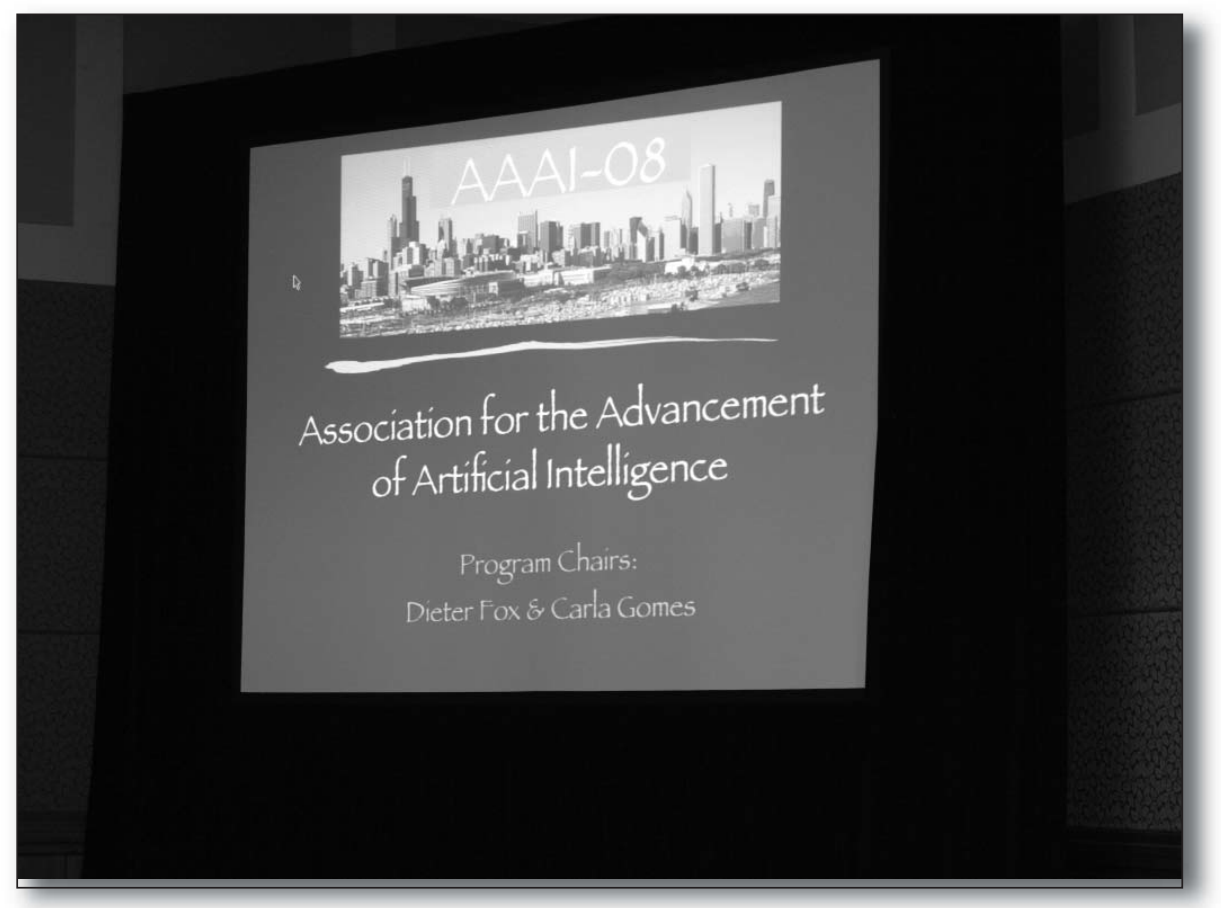

Decadal Landsat Images," an application that is being developed to help automate the selection of images from the Landsat data from 2004-2006 to construct a data map that will be distributed to the public at no charge through a U.S. Geological Survey website. The system will likely become the next generation backdrop for GoogleEarth and also assist Earth scientists by providing improved automated tools to study the Earth's changing ecosystem. More than 300,000 images must be evaluated to select the roughly 9,500 that will compose the map.

The Universidad Nacional de Asunción (Paraguay) presented "Crop Selection for Optimal Soil Planning using Multiobjective Evolutionary Algorithms," a system that aids farm managers in developing optimized plans for crop cultivation based on soil characteristics, and thus minimizes the need for soil treatment, thereby reducing the costs and potential environmental damages. The system is geared toward sustainable land use practices, with benefits that decrease rural poverty, protect watersheds, increase biodiversity, increase sustainable agricultural production, and increase food security. It employs multiobjective evolutionary algorithms using eight processors.

\section{Medical and Health Care}

Five IAAI applications were in the medical and health-care fields. Coronary heart disease (CHD), the leading cause of death worldwide, can be detected using an echocardiogram, which is difficult to interpret even for experts. Siemens and Motorola presented "Learning Sparse Kernels from 3D surfaces for Heart Wall Motion Abnormality Detection," a project that is exploring a novel automatic technique using a machine learning classifier to detect the abnormalities that indicate coronary heart disease.

McGill University (Canada) presented "Adaptive Treatment of Epilepsy via Batch-mode Reinforcement Learning" a system that utilizes machine learning techniques to optimize the deep brain stimulation strategy for the treatment of epilepsy to minimize the frequency and duration of seizures.

Traditional Chinese medicine (TCM) is an ancient medical system that still accounts for 40 percent of all health care in China. Zhejiang University (China) presented "Semantic Web Development for Traditional Chinese Medicine," a system that bridges the gaps between a plurality of legacy and heterogeneous relational databases from both TCM and West- 
ern medicine, enabling ontologybased query and search across database boundaries providing knowledge to TCM practitioners on herb and drug usage and Chinese herb-Western prescription drug interactions. The project illustrates how the semantic web can connect data across domain and community boundaries to facilitate interdisciplinary and cross-cultural studies. It also leverages the progress of biomedical informatics to address the preservation and modernization of the ancient Chinese cultural heritage of TCM.

Technische Universität München (Germany), LORIA-INRAI (France), Klinikum Rechts der Isar (Germany) presented "On-line Recognition of Surgical Activity for Monitoring in the Operating Room," a project that aims to design support systems for the operating room ranging from simple tasks such as the activation of lights to more complex tasks such as surgical procedures.

Finally, Bar-Ilan University (Israel) and the University of Haifa (Israel) presented "COACH-Cumulative Online Algorithm for Classification of Handwriting Deficiencies," an online handwriting evaluation tool for classifying and remediating handwriting deficiencies using machine learning and data mining.

\section{Resource Scheduling and Allocation}

There were three applications in the resource scheduling and allocation domain. City University of Hong Kong (Hong Kong SAR) presented "Using AI for Olympic Equestrian Event Preparation," a web-based workforce scheduling system for approximately 1,000 part-time volunteers and staff at the 2008 Beijing Olympic Equestrian Events held in Hong Kong.

The National University of Singapore presented "A Hybrid Approach to Convoy Movement Planning in an Urban City," a system that dynamically routes up to 100 military convoys in a real city road network addressing the need for a defensive strategy, dynamically replanning when changes occur (such as road congestion, enemy attacks, and so on).

Finally, the South China University of Technology (China), Red Jasper Ltd. (Hong Kong), and the Hong Kong University of Science and Technology presented "A Vehicle Routing System to Solve a Periodic Vehicle Routing Problem for a Food Chain in Hong Kong," a system that optimizes the supply delivery schedule for one of the largest food and restaurant chains in Hong Kong. Results shows that at least 14 vehicles can be saved, each valued at one million Hong Kong dollars with 0.3 million Hong Kong dollars of running cost for a total of 18.2 million annually (\$2.34 million U.S.) Savings of up to 23 percent in asset costs and annual operating costs for the food chain are forecasted.

\section{Volunteers Essential to AAAI}

Planning and preparing for such an ambitious conference was a tremendous undertaking - the product of countless volunteer hours from dedicated AAAI members. For instance, as part of the organization's mission of promoting education, a small number of students had the opportunity to chat with an AAAI fellow over an informal lunch during the conference. This provided a unique opportunity for students to meet some of the most noted and accomplished scientists in the field.

AAAI is particularly grateful for the efforts of this year's Conference Committee Chair Yolanda Gil, Technical Program Cochairs Dieter Fox and Carla Gomes, and IAAI-09 Chair Mehmet Goker and Cochair Karen Haigh. So many organizers and participants, such as special track organizers Oren Etzioni, Craig Knoblock, Pat Langley, Alan Schultz, Drew Bagnell, and Wolfram Burgard; competition chairs and cochairs Mike Genesereth, Eric Schkufza, Andrew Gilpin, David Aha, and Sebastian Thrun; and mobile robot exibition and workshop cochairs Paul Oh, Odest Chadwicke Jenkins, Youngmoo Kim, and Monica Anderson, gave generously of their time and talents.

During his acceptance speech for the AAAI Distinguished Service Award, Ronald J. Brachman, vice president, Worldwide Research Operations, Yahoo Research, noted, "It is actually an honor to serve the community." He urged the audience to follow suit: "When the opportunity for service knocks, please step up and serve the community. It is our responsibility to build and maintain the service organizations that make the field possible." The proceedings for AAAI and IAAI-08 are available in paper or CD format from AAAI Press. ${ }^{5}$

Plans are already underway for future AAAI and IAAI conferences. The next IAAI conference will be held July 11-15, 2009, in Pasadena, California. In 2010, IAAI and AAAI go to Atlanta, Georgia July 11-15, while in 2011, the two conferences will be held for the very first time in San Francisco, California, from August 7-11.

\section{Thanks to Our Generous Sponsors}

AAAI would like to thank the following organizations and individuals for their generous support of the AAAI-08 conference: Microsoft Research, National Science Foundation, Google Inc., Michael Genesereth, NASA Ames Research Center, Yahoo Research Labs, Intel, Naval Research Laboratory, University of Southern California/Information Sciences Institute, Boeing, IBM Research, Toyota Motor Engineering and Manufacturing North America Inc., Cornell University Intelligent Information Systems Institute, D. E. Shaw, and ACM/SIGART.

Notes

1. For a précis and an MP3 recording, see research.microsoft.com/ horvitz/AAAI_Presidential_Lecture_Eric_Horvitz.htm.

2. For all the results, see www.cs.ualberta. $\mathrm{ca} / \sim$ pokert/2008/results.

3. See www.general-game-playing.de/teach ing.html for results.

4. For more information on entries and details about the seven winners, please visit the competition website (www.aivideo. org).

5. See www.aaai.org/Press/Proceedings/aaai 08.php.

Sara Reece Hedberg is an independent journalist and owner of Emergent, Inc. She can be reached at sara@hedberg.com. 